Bawden, F. C. \& Pirie, N. W. (1959). J. gen. Microbiol. 21, 438-456

\title{
The Infectivity and Inactivation of Nucleic Acid Preparations from Tobacco Mosaic Virus
}

\author{
By F. C. BAWDEN and N. W. PIRIE \\ Rothamsted Experimental Station, Harpenden, Hertfordshire
}

\begin{abstract}
SUMMARY: The infectivity of nucleic acid preparations made by disrupting tobacco mosaic virus with phenol was increased, relative to that of intact virus, by keeping test plants in darkness or at $37^{\circ}$ for some time before they were inoculated. The differences in susceptibility to infection of leaves in different physiological states was too great to be explained by differences in the ability of leaf extracts to inactivate nucleic acid preparations in vitro. The spontaneous inactivation of the preparations in vitro was not prevented by inhibitors of ribonucleases and most additions to the preparations increased the rate of inactivation. Not all the inactivations are readily explicable on the assumption that the minimal infective unit is a pure nucleic acid built up solely from nucleotides. Leaf sap and saliva are reasonably assumed to inactivate because they contain ribonuclease; also, inactivations by formaldehyde phenylglyoxal, and thiaminase in the presence of thiamine, may well reflect reactions with known components of nucleic acids. However, it is less easy to invoke such actions to explain the inactivation by spermine, 'interferon', some oxidizing agents, leaf mitochondria in the presence of some other substances, and the greater rate of inactivation in vacuo than in air. Although nucleic acid seems essential for infectivity, it seems prudent to suspend judgement about the precise chemical identity of the minimal infective unit.
\end{abstract}

Some treatments have long been known to inactivate tobacco mosaic virus (TMV) without appreciably affecting its physical or serological properties (Bawden \& Pirie, 1937). While the nucleoprotein particles were thought to be the minimal infective units, this phenomenon remained unexplained, but the discovery that virus fragments consisting predominantly of nucleic acid can be infective (Gierer \& Schramm, 1956; Fraenkel-Conrat, 1956) provided an obvious explanation: these treatments presumably have little effect on the surface protein, which is what mainly determines physical and serological properties, but affect more deeply buried structures.

Virus fragments soon lose infectivity in conditions in which the nucleoprotein is stable; a structure essential for reproduction is, therefore, protected in intact particles from many potentially inactivating treatments. A fuller knowledge of the conditions in which TMV fragments become inactivated might throw light on the roles of the different components of virus particles and might help to identify more closely the minimal infective unit. That nucleic acid is an essential part of this unit seems established, but that it alone is infective remains unproven. Preparations of nucleic acid are so much less infective than intact virus containing the same amount of $P$ that analytical studies on the preparations have little bearing on the problem. There is no advantage in postulating additional components gratuitously, but if infectivity is small because preparations are contaminated with much non-infective nucleic 
acid, the infectivity of those particles that are infective could well depend on components other than those indicated by the conventional nucleic acid formula. If all the original virus particles were infective, inert nucleic acid could arise only because of inactivation during the course of its separation; if this happens, different methods of disrupting the virus might be expected to yield products with different activities. But preparations made by heating, or by exposure to heat or detergents, all have similar infectivities.

An alternative explanation of the disparity between the infectivity of TMV and of nucleic acid preparations is provided by the sensitivity of the nucleic acid towards agents that it meets during the processes of infection. This explanation suggests that infectivity might be stabilized by some treatment or addition, and the reported increase in infectivity when the nucleic acid and protein moieties are recombined (Fraenkel-Conrat \& Singer, 1957) may be an example of such a treatment. However, other explanations are possible, for virus protein could also obviously be adding some component necessary for infectivity. To find something other than virus protein that will increase infectivity, whether by stabilizing the fragments in vitro or by protecting them from hazards on or in leaves, it is necessary to know more about inactivating conditions so that these can be avoided. We (Bawden \& Pirie, 1957 b) suggested that ribonuclease in the nucleic acid preparations might be the cause of their instability, but inhibitors of this enzyme have not stabilized the preparations and we have had inactivation in many circumstances when it would be unreasonable to invoke ribonuclease as the cause. Indeed, we have found no in vitro treatment that increases infectivity and our tests have served only to increase the variety of treatments that destroy it. Current knowledge on the structure of nucleic acid provides no immediately obvious explanation for some of these.

The relative infectivities of nucleic acid preparations and of intact TMV can be changed by changing the conditions under which plants are kept before they are inoculated, for infection by nucleic acid preparations is more affected by the physiological condition of the test plants than is infection by intact virus. This suggests that nucleic acid rarely manifests full infectivity, presumably because much potentially infective material is inactivated in the inoculated leaves. Whether plants are susceptible or resistant, their leaves contain a variety of systems able to inactivate virus fragments and so make unlikely their survival during the necessary interval between inoculation and virus multiplication. Some extracts from plants resistant to infection by nucleic acid inactivated more than did comparable extracts from susceptible plants, but this was not always so. Most of the fragments, therefore, may be potentially infective but be prevented from showing it. If this is the correct interpretation of the relatively low infectivity of nucleic acid preparations, analytical evidence that a 'pure' nucleic acid can infect becomes more cogent in arguments about minimal infective units, but until the interpretation is better established such evidence needs to be used cautiously. For brevity the term nucleic acid will be used in this paper to mean a preparation consisting mainly but not necessarily exclusively of nucleic acid. 


\section{METHODS}

The TMV preparations used were all made from systemically infected tobacco leaves by a method that does not wholly eliminate ribonuclease but decreases it to a barely detectable level (Pirie, 1956). The virus was precipitated by bringing clarified sap to $\mathrm{pH} \mathrm{3 \cdot 3}$, redissolving it and precipitating with ammonium sulphate ( $\frac{1}{3}$ saturation). After repeating the precipitations with acid and salt, the preparation was incubated for a day at $37^{\circ}$ in $0.1 \mathrm{M}$-citrate ( $\mathrm{pH} 6.5$ ), and again precipitated with ammonium sulphate. It was then dissolved to give a solution containing 10-15 g./l. from which the virus was sedimented three times at $50,000 \mathrm{~g}$.

In most of the experiments reported here, nucleic acid made by the modification of the phenol method (Gierer \& Schramm, 1956) that we have already described (Bawden \& Pirie, 1957 $b$ ) was used. Results with nucleic acid made by fission with warm detergent (Fraenkel-Conrat, 1956) are specifically noted. The great advantage of the phenol method over either the detergent or heating method (Knight, 1956) is that it leaves no intact TMV to be removed by ultracentrifugation. We have not compared products from the three methods in great detail, but they differ no more in infectivity than do separate preparations made with phenol. Usually a large batch was made at one time, divided into many small samples in individual tubes, 'and stored at $-15^{\circ}$, when infectivity is retained for many months. After being kept frozen for some weeks, preparations were often less infective immediately after thawing than they were after keeping for a few hours at $20^{\circ}$ or $1 \mathrm{hr}$. at $37^{\circ}$. This effect, however, was not always obtained, and the stability of different preparations when kept at $20^{\circ}$ or $37^{\circ}$ also varied somewhat. With all there was much infectivity remaining after $2-3 \mathrm{hr}$. at $37^{\circ}$, or $20 \mathrm{hr}$. at $20^{\circ}$, and at $0^{\circ}$ there was no obvious inactivation during $20 \mathrm{hr}$. The preparations were thawed and kept for some hours before they were used in experiments, and samples were usually kept at $0^{\circ}$ to act as controls in experiments involving exposure to $37^{\circ}$. Each preparation of nucleic acid was tested to ensure that it was free from residual TMV, by showing that infectivity was rapidly and completely destroyed by incubation with leaf ribonuclease.

Infectivity assays were made in Nicotiana glutinosa $\mathrm{L}$. To increase their susceptibility in the summer, plants were raised in shaded glasshouses, and they were usually put in darkness or at $37^{\circ}$ for the $24 \mathrm{hr}$. immediately before they were inoculated. Eight treatments were usually compared in each test, and each was inoculated to at least 9 half leaves. The treatments were distributed systematically over the test plants to compensate as far as possible for differences in susceptibility between different plants and between leaves occupying different positions on the stems. The need for this is even greater when using nucleic acid preparations as inocula than when using intact TMV, for the number of lesions produced by such preparations vary much more from leaf to leaf even when the leaves look similar. We (Bawden \& Pirie, 1957b) reported that young plants are relatively more susceptible to infection by virus fragments than by TMV and that the upper leaves of plants are relatively more 
susceptible than the lower ones. This second statement is true when plants are grown in normal glasshouse conditions during summer, but by no means always holds with plants growing in the winter or under shade in summer. Then all leaves may be of comparable susceptibility, or the older ones may be the more susceptible to infection by nucleic acid as they nearly always are when the inoculum is TMV.

Samples under test were usually incubated as $\mathbf{0 . 1} \mathrm{ml}$. lots containing $\mathbf{2 \cdot 5}-$ $5 \mu \mathrm{g}$. P. They were diluted to $1 \mathrm{ml}$. with water before inoculating to Nicotiana glutinosa, which was done by rubbing the upper surfaces of the leaves with the forefinger wet with inoculum. During the time taken (1-2 min.) to inoculate one sample to 9-12 half leaves, there was no measurable inactivation of the sample, for on average of many counts the last rubbed leaves produced as many lesions as the first. However, after dipping the forefinger in such samples, inoculation must not be too long delayed, for within an hour of a protracted dip of $1 \mathrm{~min}$. the infectivity of samples may be halved, presumably by something secreted through the skin.

\section{RESULTS}

\section{Condition of plants and relative susceptibility to $T M V$ and nucleic acid preparations}

The number of lesions produced by a given inoculum of TMV varies with the physiological state of the plants. Young, rapidly growing plants produce more lesions than old, slowly growing ones, and plants grown in shade produce more than those grown in bright light; also, with otherwise similar plants, susceptibility to infection is increased by keeping them in darkness or at high temperature for a day or two before they are inoculated (Bawden \& Roberts, 1947, 1948; Kassanis, 1952). Differences in the physiological state of test plants have an even greater effect on the numbers of lesions produced by nucleic acid preparations. Table 1 shows that, whereas keeping in darkness or at $37^{\circ}$ for two days doubled the numbers of lesions produced by TMV, it increased the number produced by nucleic acid preparations by a factor of 10 or more.

Dilution affects the infectivity of the two kinds of inocula differently. The number of lesions produced by TMV decreases with dilution by less than the factor of dilution, whereas with nucleic acid preparations the numbers fall more steeply. The relative infectivity of the two types of inocula/unit of $P$, therefore, is not a fixed quantity but depends greatly on the state of the test plants and the concentrations at which the two are compared, and the nucleic acid seems increasingly infective as the susceptibility of plants and the concentration of the inoculum are increased. Plants of different ages, or raised under different light regimes, show even greater differences in their susceptibility to the two inocula than those shown in Table 1. With differently treated plants that differ in their susceptibility to infection by TMV by factors of up to 10 , the more susceptible may give hundreds of lesions with a nucleic acid inoculum that produces few or none in the more resistant plants. Even with 
the most favourable conditions, however, the nucleic acid preparations have never been more than about $1 / 10$ as infective/unit of $P$ as the parent virus.

The factors that control the number of lesions an inoculum produces in a leaf can be grouped into four categories: (1) the number and type of entry points made by rubbing the leaf during inoculation; $(2)$ the ability of the inoculum to withstand inactivation by components of the leaf with which it comes in contact before reaching the site at which it can multiply; (3) the number of potential infection sites; (4) the number of potentially infective particles in the inoculum needed to initiate infection at a site. The number of entry points is unlikely to be affected by the nature of the inoculum, though it well may be by the pre-treatment of the host. Existing knowledge about the relative stability of TMV and its fragments in vitro make it probable that the two types of inoculum will differ greatly in sensitivity in the interval between entering the leaf and initiating infection, but there is less basis of confidence about effects of the other two factors.

\section{Table 1. Effect of preconditioning test plants on the relative infectivity of $T M V$ and $T M V$ nucleic acid}

The TMV and TMV nucleic acid at the specified concentration were inoculated to opposite halves of the same leaves. The control plants were left in normal glasshouse conditions and the treated ones were at $37^{\circ}$ or in darkness for the 2 days before they were inoculated; afterwards they were all put in the glasshouse. The results are expressed as the \% of the number of lesions produced by the half-leaves of the control plants inoculated with TMV at 5 mg./l.; the actual numbers produced by these 12 half-leaves were 512 and 620 .

\begin{tabular}{ccccc}
\multicolumn{5}{c}{ Treatment of plants } \\
Inoculum & Control & 2 days at $37^{\circ}$ & Control & $\begin{array}{c}\text { 2 days in } \\
\text { darkness }\end{array}$ \\
TMV 5 mg./l. & 100 & 160 & 100 & 160 \\
1 & 43 & 79 & 28 & 45 \\
$0 \cdot 2$ & 15 & 4.2 & 3.5 & 9 \\
RNA 5 mg.P/l. & 20 & 152 & 4 & 64 \\
1 & 3 & 35 & 1.5 & 15 \\
0.2 & $0 \cdot 4$ & 10 & 0.3 & 3
\end{tabular}

Kleczkowski (1950) challenged the generally accepted idea that infection results from chance encounters between single virus particles and infection sites of uniform susceptibility, and our results with the two types of inocula seem compatible only with the idea that individual infection sites differ qualitatively. For instance, some allow infection readily by either TMV or nucleic acid, others only with TMV, and changes in the condition of leaves affect behaviour to the two inocula differently. Also, the greater effect of dilution on nucleic acid preparations is apparently not a consequence of inactivation by simple dilution, because samples kept diluted for some hours are no less infective than samples left concentrated and then diluted immediately before inoculation. Hence the chances of a potentially infective dose of nucleic acid infecting at a given site are increased by increasing the total amount of nucleic acid applied, and extra nucleic acid to some extent simulates the effect of combination with virus protein. This again could have two 
explanations; increasing the amount of nucleic acid may simply increase the chances that one infective unit will survive and infect, or to start the processes that lead to virus multiplication, different numbers of infective units may be needed at different sites. Increasing the total concentration of nucleic acid in inocula by adding yeast nucleic acid to TMV nucleic acid did not increase the number of lesions produced.

\section{Inactivation by systems of the type encountered during inoculation}

We have not tried to define the nature of the change in leaves kept at $37^{\circ}$ or in darkness that increases their susceptibility to infection, but we have compared various types of extracts from leaves of old and young plants for their ability to inactivate nucleic acid preparations in vitro. These comparisons have not explained the differences in susceptibility, but they have shown that there are so many inactivating components in leaves that it becomes increasingly surprising that nucleic acid should ever be infective.

Even before it enters a cell, nucleic acid is at some risk. Water that has lain for $10 \mathrm{~min}$. on an undamaged Nicotiana glutinosa leaf as a pool 2-3 mm. deep will inactivate nucleic acid in $4 \mathrm{hr}$. at $37^{\circ}$. This inactivating power is stable for a month at $0^{\circ}$ but is lost on boiling. It has probably little influence on the course of infection because it is not shown unequivocally at room temperature and the potency of washes from leaves of different type is not obviously correlated with their susceptibility to infection. It may be a manifestation of ribonuclease activity but this cannot be demonstrated with certainty, because the washes act strongly only when undiluted and the ability to inactivate nucleic acid is much the most sensitive of the tests for ribonuclease.

Ribonuclease occurs in all the leaf saps that have been studied; it is therefore an obvious potential inactivating agent and it seemed possible that variation in the amount of enzyme in Nicotiana glutinosa leaves might account for differences in their susceptibility. Many comparisons, with yeast nucleic acid as substrate, have shown that sap from older, resistant leaves has two to three times the enzyme activity of sap from younger, susceptible leaves. This is a trend in the opposite direction from that found in pea leaves (Pisum sativum, Holden \& Pirie, 1955) but it is in the direction corresponding to the differences in susceptibility. Quantitatively however the variation in enzyme activity is inadequate to explain the differences in susceptibility unless some ad hoc postulates are made about the distribution of ribonuclease in cells of different age.

From its ribonuclease content alone Nicotiana glutinosa sap could be expected to inactivate nucleic acid. Table 2 shows that it does, that the extent of inactivation depends on the dilution of the sap and the temperature of the mixture, and that heating sap diminishes its activity. Other similar experiments showed that activity is lost on more prolonged heating at $100^{\circ}$ but not on incubation, in the presence or absence of air, at $37^{\circ}$ for several days. These results are compatible with ribonuclease action. Diluted sap and ribonuclease (leaf or pancreatic) solutions, with similar abilities to hydrolyse yeast nucleic acid, differed in their ability to inactivate nucleic acid to an extent that 
seemed to exceed the experimental error. The greater inactivating ability of sap prompted a search for a method of distinguishing between inactivation brought about by ribonuclease and other systems. Although this did not succeed in its main object, it showed that differences in susceptibility are not consistently related to the properties that we have measured in vitro.

\section{Table 2. The inactivation of nucleic acid by sap}

Sap from Nicotiana glutinosa was made as described in the text and one sample was heated for $5 \mathrm{~min}$. at $80^{\circ}$ and another at $100^{\circ}$. Suitable volumes were then mixed with water to give $0.1 \mathrm{ml}$. at the specifled dilution and mixed with $0.01 \mathrm{ml}$. of nucleic acid solution containing $50 \mathrm{mg}$. P/l. After $15 \mathrm{~min}$. at the stated temperatures, the mixtures were diluted to $1 \mathrm{ml}$. and inoculated to $N$. glutinosa.

Nucleic acid mixed with

Water

Sap 1/12.5

Sap $1 / 50$

Sap 1/200

Sap $1 / 50$ heated at $80^{\circ}$

Sap $1 / 50$ heated at $100^{\circ}$

Sap $1 / 50$

Sap $1 / 200$

\begin{tabular}{|c|c|}
\hline $\begin{array}{l}\text { Temperature of } \\
\text { inoculation }\left({ }^{\circ}\right)\end{array}$ & $\begin{array}{l}\text { Average } \\
\text { number of } \\
\text { lesions/leaf }\end{array}$ \\
\hline 18 & 41 \\
\hline 0 & $\mathbf{0}$ \\
\hline 0 & 0.5 \\
\hline o & 17 \\
\hline 0 & 1 \\
\hline 0 & 14 \\
\hline 18 & $\mathbf{0}$ \\
\hline 18 & 2 \\
\hline
\end{tabular}

Table 3. Inactivation of nucleic acid by sap from leaves of susceptible and resistant leaves

Sap from susceptible and resistant leaves of Nicotiana glutinosa plants was diluted to the extent specifled when mixed with the nucleic acid solution. After incubation for the time and at the temperature stated, the mixtures were diluted $1 / 10$ and inoculated to $N$. glutinosa. Leaves on the resistant plants gave no lesions with a nucleic acid preparation that produced more than 200 lesions/leaf on the susceptible plants.

\begin{tabular}{|c|c|c|c|}
\hline \multirow[b]{2}{*}{ Origin and dilution of sap } & \multicolumn{2}{|c|}{ Incubation } & \multirow{2}{*}{$\begin{array}{c}\text { Average } \\
\text { number of } \\
\text { lesions/leaf }\end{array}$} \\
\hline & $\begin{array}{c}\text { Temperature } \\
\left({ }^{\circ}\right)\end{array}$ & $\begin{array}{c}\text { Time } \\
\text { (min.) }\end{array}$ & \\
\hline Resistant leaf $\quad 1 / 50$ & o & 10 & 38 \\
\hline Susceptible leaf $1 / 3$ & $\mathbf{0}$ & 10 & 32 \\
\hline $1 / 12$ & $\mathbf{0}$ & 10 & 95 \\
\hline $1 / 50$ & $\mathbf{0}$ & 10 & 128 \\
\hline Resistant leaf $1 / 500$ & 18 & $\mathbf{5 0}$ & 4 \\
\hline Susceptible leaf $1 / 500$ & 18 & $\mathbf{5 0}$ & 29 \\
\hline $1 / 3$ & Mixed imme & ly before & 153 \\
\hline Control in water & 18 & - & 240 \\
\hline
\end{tabular}

During the summer, results of the types set out in Table 3 are typical. Sap from highly resistant leaves inactivates nucleic acid five to ten times as rapidly as does sap from susceptible leaves. This factor is probably outside the combined possible uncertainties of the enzyme assays, which showed the sap from resistant plants to contain twice as much activity as that from susceptible plants, and the infectivity tests. There is therefore good reason to think that at least sap from resistant leaves contains an inactivating mechanism in addition to ribonuclease. But even a tenfold difference in inactivating power does not go 
very far towards explaining the observed differences in susceptibility. Furthermore, although young winter-grown Nicotiana glutinosa plants also give many more lesions than old plants when inoculated with the same nucleic acid solution, the ability of sap from old and young leaves in winter to inactivate nucleic acid differs little. Indeed, sap from susceptible leaves was sometimes more potent.

Although the ability of sap to inactivate nucleic acid is largely destroyed by boiling, which suggests that it depends on enzyme-like components, tests with sap dialysates were made because nucleic acid is inactivated by some substances of low molecular weight. All dialysates tested were effective, but as they had only $1 / 10$ to $1 / 100$ the activity of the sap it is unlikely that this type of inactivation can explain differences in susceptibility.

The sap used in the experiments we have described so far was made by grinding one or two half-leaves in a mortar with twice their weight of water (the other half was left on the plant and tested for susceptibility) and centrifuging for $20 \mathrm{~min}$. at $10000 \mathrm{rev} . / \mathrm{min}$. after lying for $2 \mathrm{hr}$. at room temperature. This ageing ensures that chloroplast fragments, mitochondria and similar structures aggregate so that they can be centrifuged out. No experiments were made with these structures from Nicotiana glutinosa leaves because they are not a suitable source of enzymically active preparations of mitochondria. Active mitochondrial preparations can be made by grinding young tobacco (N. tabacum) leaves at $0^{\circ}$ in buffered $0 \cdot 4 \mathrm{M}$-sucrose solution and fractionating the extract by differential centrifugation (Pierpoint, 1959). These are still contaminated with chloroplast fragments but examination under the microscope suggests that mitochondria make up about half the material present. They are therefore very different from the crude preparations, made from old infected tobacco leaves, which Bawden \& Pirie (1957 a) found inactivated the Rothamsted culture of tobacco necrosis and some other viruses.

When used sufficiently concentrated, or at $37^{\circ}$, preparations of mitochondria inactivate nucleic acid, probably because they carry a trace of ribonuclease activity. It is of more interest that they also carry enzymes involved in the tricarboxylic acid cycle (Pierpoint, 1959); by analogy with mammalian mitochondria they probably also carry enzymes involved in other syntheses. Some of these are probably integral parts of the mitochondrion but others may well have been absorbed during the processes of fractionation (cf. Pirie, 1959). By choosing conditions in which mitochondria alone have relatively little effect on nucleic acid, the effect of various substrates or stimulants can be studied. Table 4 shows that maleate and ethylenediaminetetraacetate (EDTA) greatly enhance inactivation by the mitochondria, although alone they have little inactivating effect. The effect of citrate is equivocal but some other substrates, notably $\alpha$-ketoglutarate and isocitrate also promote inactivation by many preparations of mitochondria.

These substances may act in various ways. The tricarboxylic acid cycle substrates, for example, may be converted into substances that act directly on the nucleic acid, whereas EDTA, which is unlikely to act as a substrate, may disorganize mitochondria and release an inactivating agent. To test 
this last possibility we examined the effect on nucleic acid of supernatant fluids made by centrifuging at $10000 \mathrm{~g}$ samples of a mitochondrial preparation (10 mg. N/l.) that had been treated in different ways. After $12 \mathrm{hr}$. in water at $0^{\circ}$ the supernatant fluid diminished the infectivity of nucleic acid to about half in $2 \mathrm{hr}$. at $20^{\circ}$, but the diminution was to less than a tenth when 1 g. EDTA/l. replaced the water. At this concentration EDTA alone does not inactivate. Mitochondria, as has been said, carry a trace of ribonuclease activity in spite of careful fractionation, and this is perhaps stimulated, or more may be liberated, by EDTA. To get significant hydrolysis of yeast nucleic acid it is necessary to use suspensions of mitochondria 200 times more concentrated than those used to inactivate nucleic acid and this hydrolysis is not stimulated by EDTA. It is therefore unlikely that ribonuclease is the inactivating agent but a more detailed study of this phenomenon is not worth while until more is known about leaf mitochondria.

\section{Table 4. The effect of various agents on the inactivation of nucleic acid by leaf mitochondria}

Mitochondria, made from Nicotiana tabacum leaves as described in the text, were suspended at pH 6 at the concentration, and in the fluid, specified, mixed with nucleic acid and the mixture diluted and inoculated to $N$. glutinosa after $2 \mathrm{hr}$. at $18^{\circ}$.

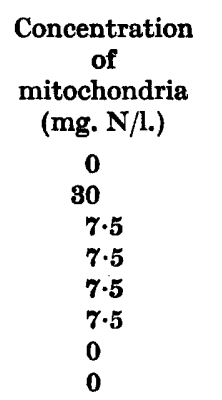

$\begin{array}{lc} & \begin{array}{c}\text { Average } \\ \text { number } \\ \text { of } \\ \text { lesions/leaf }\end{array} \\ \text { Suspending fluid } & 50 \\ \text { water } & 4 \\ \text { water } & 25 \\ \text { water } & 2 \\ \text { 2 g. EDTA/l. } & 15 \\ \text { 0.02 M-citrate } & 0.5 \\ \text { 0.02M-malate } & 26 \\ \text { 2 g. EDTA/l. } & 20 \\ \text { 0.02M-maleate } & \end{array}$

Inactivation by some enzyme, or enzyme-like, systems

Some of these results, especially those with mitochondria, suggest that the infectivity of nucleic acid depends on some component or structure other than those in the conventional nucleic acid formula. The range of agents that could profitably be tried as inactivators is immense, and it is probable that the use of relatively specific enzyme preparations will provide evidence about the actual nature of the infective agent. This has happened in the past, for the first evidence that protein was a part of potato virus $X$ particles came from a study of the action of proteases (Bawden \& Pirie, 1936). Accessibility and general interest have controlled our choice of enzyme preparations with which to start.

Thiaminase. This enzyme is found in Pteridophytes, moulds and bacteria, but so far in only one higher plant. Its ability to catalyse transfer reactions between various heterocyclic compounds related to thiamine (vitamin $B_{1}$ ) is well known but, in the course of reviewing the literature on the enzyme, 
Kenten $(1957$ a) mentioned that it catalyses an action between thiamine and some purines and pyrimidines. This suggests that its normal action in vivo may be more general and involve substitutions on nucleic acids.

Table 5 shows that a preparation of thiaminase made from bracken leaves by Kenten $(\mathbf{1 9 5 7} b)$ had little inactivating action on its own, but inactivated rapidly in the presence of thiamine. For this action thiamine cannot be replaced by either yeast or thymus nucleic acids, pyridine, piperidine or a number of other bases; nor does thiamine increase the rate of inactivation of the nucleic acid by unfractionated leaf sap, mitochondria or salivary ribonuclease. It would be premature to claim that this effect is caused by thiaminase, but it is clearly not caused by a nuclease in the normal sense of that term.

Table 5. The effect of thiaminase and thiamine on the infectivity of nucleic acid

Fach tube contained the stated volume of thiaminase solution, in a final volume of $0.1 \mathrm{ml}$., along with thiamine and nucleic acid. After $4 \mathrm{hr}$. at $18^{\circ}$ they were diluted to $1 \mathrm{ml}$. and inoculated to Nicotiana glutinosa.

$\begin{array}{ccc}\begin{array}{c}\text { Volume of } \\ \text { thiaminase } \\ \text { solution (ml.) }\end{array} & \begin{array}{c}\text { Concentration } \\ \text { of thiamine } \\ \text { (g./l.) }\end{array} & \begin{array}{c}\text { Average } \\ \text { number of } \\ \text { lesions/leaf }\end{array} \\ 0.01 & 8 & 2 \\ 0.01 & 2 & 1 \\ 0.01 & 0.5 & 18 \\ 0.01 & 0.125 & 39 \\ 0.01 & 0 & 76 \\ 0.003 & 2 & 3.5 \\ 0 & 8 & 40 \\ 0 & 0 & 85\end{array}$

Saliva. The presence of many enzymes in this secretion is generally recognized but little attention seems to have been paid to its ribonuclease. The enzyme is important here for two reasons : first to show what hazards a sensitive nucleic acid faces from pipetting and the labelling of tubes, and secondly because a comparison of the inactivating power of saliva and purified pea or pancreatic ribonuclease, with equal ability to hydrolyse yeast nucleic acid, could give evidence about the number of different enzymes that can inactivate. Saliva diluted $1 / 200$ caused $90 \%$ inactivation in $40 \mathrm{~min}$. and complete inactivation in $3 \mathrm{hr}$. In that experiment there was the same inactivation with pea ribonuclease at 0.01 units $/ \mathrm{ml}$. (Holden \& Pirie, 1955) and this was the ratio of their activities measured by the hydrolysis of yeast nucleic acid. There is therefore no reason to look for enzymes other than ribonuclease to explain the action of saliva.

Interferon. Isaacs \& Lindenmann (1957) gave the name interferon to an extract made from chick embryo that had been exposed to inactivated influenza virus. It inhibits the multiplication of several animal viruses but it neither inactivates intact TMV nor inhibits infection by it. The ribonuclease activity of concentrated solutions is barely perceptible with yeast nucleic acid as substrate but they inactivate TMV nucleic acid when diluted 1/1000. Table 6 shows this and that some of the activity withstands boiling. It is difficult to 
attribute this action to a ribonuclease, and it may not be an enzyme action, but other experiments showed that the extent of inactivation increased with time and it proceeded more rapidly at $18^{\circ}$ than at $0^{\circ}$. The preparations may contain more than one component able to act on the nucleic acid, and we have no evidence that any of these is the substance that interferes with the multiplication of animal viruses.

\section{Table 6. Inactivation of nucleic acid by interferon}

Nucleic acid ( $8 \mathrm{mg} . \mathrm{P} / \mathrm{l}$.) was exposed to the stated dilutions of a preparation of interferon for $1 \mathrm{hr}$. at $18^{\circ}$, when the solutions were diluted and inoculated to Nicotiana glutinosa.

$\begin{array}{cc}\begin{array}{c}\text { Dilution of } \\ \text { interferon }\end{array} & \begin{array}{c}\text { Average number } \\ \text { of lesions/leaf }\end{array} \\ 1 / 10 & 0 \\ 1 / 100 & 2 \\ 1 / 1000 & 43 \\ 1 / 10,000 & 54 \\ \text { Boiled interferon } & 16 \\ \text { at } 1 / 100 & \\ \text { Water } & 158 \\ & \\ & \\ & \\ & \end{array}$

The surface of leaf sap becomes brown in a few minutes when it is exposed to air at room temperature. This can be prevented by cooling, excluding air, or adding inhibitors or reducing agents. The normal conditions of inoculation expose exudate from damaged leaf cells generously to oxidation; obviously, this may damage an infecting agent and might account for the low infectivity of nucleic acid. In an attempt to minimize this risk and protect the inoculum during entry, a few leaves were inoculated at $0^{\circ}$ or in $\mathbf{N}_{2}$, but there was no striking effect on the number of lesions produced. A parallel attempt to protect the inoculum by adding neutralized ascorbic acid or cysteine to it showed that these agents diminished rather than increased the apparent infectivity. This, together with the inactivation by leaf dialysates, prompted a study of various oxidizing and reducing systems, though it is unlikely that those studied are actually responsible for the differences in the infectibility of leaves in different physiological states.

Ascorbic acid. At concentrations within the physiological range, ascorbate is not a powerful inactivating agent and it is necessary either to expose nucleic acid to it at $37^{\circ}$ or to use prolonged exposures at room temperature. Table 7 shows this, and it is clear that the inactivation can be prevented, or at any rate decreased, by excluding air and by adding diethyldithiocarbamate which inhibits copper-catalysed oxidations. Other experiments showed that the extent of inactivation increases with time and that nucleic acid is not quickly inactivated when added to ascorbate that had undergone aerobic oxidation. These phenomena suggest that the actual inactivating agent is neither ascorbic acid nor its oxidation product, dehydrascorbic acid, but something, presumably an unstable oxidizing agent, produced during the oxidation.

Glutathione and iron. When tested on nucleic acid made by disrupting TMV with sodium dodecyl sulphate, glutathione behaved like ascorbic acid. In 
$3 \mathrm{hr}$. at $18^{\circ}$ there was complete inactivation by $0.01 \mathrm{M}$, and $80 \%$ inactivation by $0.002 \mathrm{M}$ solutions, in air, whereas in $\mathbf{N}_{2}$ the extent of inactivation was diminished to $\mathbf{7 0} \%$ and $\mathbf{2 0} \%$. Nucleic acid made by phenol fission is not systematically inactivated by glutathione under similar conditions. This difference may well arise because of differences in the other components of the system and it is so unphysiological that it has not seemed worth while to explore fully. But attempts to get, by adding $\mathrm{FeSO}_{4}$, conditions in which glutathione would inactivate systematically showed that $\mathrm{Fe}$ itself had a surprising ability to inactivate nucleic acid. Thus there is complete inactivation at $20^{\circ}$ in $2 \mathrm{hr}$. with $0.1 \mathrm{mM} \mathrm{Fe}$, and extensive inactivation with $0.02 \mathrm{~mm}$. Exclusion of air diminishes the inactivation and it is diminished by EDTA or $0.02 \mathrm{M}$-citrate. The amount of $\mathrm{Fe}$ in a leaf extract will depend on the extent to

Table 7. The effect of various environments on inactivation of nucleic acid by ascorbic acid

In two separate experiments, nucleic acid $(5 \mathrm{mg} . \mathrm{P} / \mathrm{l}$.) was incubated with the stated concentrations of ascorbate at $\mathrm{pH} 6$, with and without air and diethyldithiocarbamate (DIECA); after the stated time the mixtures were diluted and inoculated to Nicotiana glutinosa.

\begin{tabular}{|c|c|c|c|c|}
\hline \multicolumn{2}{|c|}{ Incubation } & \multirow[b]{2}{*}{$\begin{array}{l}\text { Concentration } \\
\text { of ascorbate }\end{array}$} & \multirow[b]{2}{*}{ Ambience } & \multirow[b]{2}{*}{$\begin{array}{c}\text { Average } \\
\text { number of } \\
\text { lesions/lea }\end{array}$} \\
\hline $\begin{array}{c}\text { Temperature } \\
\left({ }^{\circ}\right)\end{array}$ & $\begin{array}{l}\text { Time } \\
\text { (hr.) }\end{array}$ & & & \\
\hline 18 & 7 & o & Air & 52 \\
\hline 18 & 7 & $0.01 \mathrm{M}$ & Air & 2 \\
\hline 18 & 7 & $0.01 \mathrm{M}$ & $\mathbf{N}_{2}$ & 47 \\
\hline 18 & 7 & $0.01 \mathrm{M}$ & 1 mM DIECA & 71 \\
\hline 18 & 7 & $\mathbf{0}$ & $\begin{array}{c}1 \text { mM DIECA } \\
\& \text { air }\end{array}$ & 32 \\
\hline $\mathbf{0}$ & $2 \cdot 5$ & $\mathbf{0}$ & Air & 175 \\
\hline 37 & $2 \cdot 5$ & 0 & Air & 104 \\
\hline 37 & $\mathbf{2 \cdot 5}$ & $0.01 \mathrm{M}$ & Air & o \\
\hline 37 & $\mathbf{2 \cdot 5}$ & $0.002 \mathrm{M}$ & Air & 0 \\
\hline 87 & 2.5 & 0 & $\mathbf{N}_{2}$ & 95 \\
\hline $\mathbf{8 7}$ & $2 \cdot 5$ & $0.01 \mathrm{M}$ & $\mathbf{N}_{2}$ & 4 \\
\hline 37 & $\mathbf{2 \cdot 5}$ & $0.002 \mathrm{M}$ & $\mathbf{N}_{2}$ & 63 \\
\hline
\end{tabular}

which the chloroplasts have been damaged, for they contain most of the leaf Fe. Furthermore, most of the $\mathrm{Fe}$ is in organic combination. Published figures for the total Fe content of leaves and leaf fractions (e.g. Hill \& Lehmann, 1941) suffice to show only that $0.1 \mathrm{~mm}$ would be a high but not impossible value. A few measurements of the colour given by Nicotiana glutinosa sap or its dialysate with $o$-phenanthroline show the presence of not more than $0.02 \mathrm{~mm}$ $\mathrm{Fe}$ in the inorganic state. Preparations of the intact virus contain about 10 parts of Fe per million (Loring, Al-Rawi \& Fujimoto, 1958), but even if all this appears in the separated nucleic acid, solutions of the concentration we used would contain only $2 \mu \mathrm{M} \mathrm{Fe}$. Again, therefore, we have an equivocal situation. The nucleic acid may contain $\mathrm{Fe}$, but at a level only just relevant to its instability in vitro, and the level in leaf extracts is only just relevant to the instability in vivo. 
Hydrogen peroxide. As already mentioned, the fact that ascorbic acid inactivates nucleic acid while it is being oxidized suggests that there is an active intermediate and peroxide is the most obvious possibility. At $20^{\circ}$ it inactivates intact TMV in a few hours at $5 \mathrm{~mm}$, but in similar conditions $0.5 \mathrm{mM}$ inactivates nucleic acid completely and $80 \%$ of the infectivity is destroyed by $0.1 \mathrm{~mm}$. There is no measurable inactivation when the nucleic acid and $\mathrm{H}_{2} \mathrm{O}_{2}$ are mixed and tested within a few minutes, for the process needs an hour or more; if therefore some similar oxidizing agent is being produced by the other inactivating systems, there is, from experiments of this type, no means of knowing whether the rate of production, the rate of attack, or both are being measured.

Exclusion of air. Excluding air prevents or stops inactivation by the potentially oxidizing systems so far described, but with weaker inactivating agents, or when conditions are so arranged that substantial inactivation demands many hours' exposure, especially at $30^{\circ}$, inactivation is usually greater in samples from which air is excluded. We do not understand what essential factors control this surprising phenomenon; it has been encountered with many different preparations of nucleic acid. Table 8 shows the phenomenon clearly; both with and without citrate, nucleic acid was relatively stable in air at $30^{\circ}$ but inactivated when air was excluded. Similarly, at $37^{\circ}$, inactivation was complete without air and partial in air.

Table 8. The effect of excluding air on the stability of nucleic acid

Solutions of nucleic acid ( $5 \mathrm{mg}$. P/l.) in the environment stated were left for $12 \mathrm{hr}$. before they were diluted and their infectivities compared.

\begin{tabular}{|c|c|c|c|}
\hline Nucleic acid in & Temperature $\left({ }^{\circ}\right)$ & Atmosphere & $\begin{array}{l}\text { Number of } \\
\text { lesions on } \\
\text { eight half } \\
\text { leaves }\end{array}$ \\
\hline 0.02 M-citrate (pH 6) & $\left\{\begin{array}{r}0 \\
37 \\
30 \\
30 \\
30\end{array}\right.$ & $\begin{array}{l}\text { Air } \\
\mathbf{N}_{2} \\
\mathbf{N}_{2} \\
\text { Air } \\
\mathbf{N}_{2}\end{array}$ & $\begin{array}{c}\mathbf{5 2} \\
\mathbf{0} \\
\mathbf{0} \cdot \mathbf{5} \\
49 \\
0\end{array}$ \\
\hline Water & $\left\{\begin{array}{l}30 \\
87 \\
37\end{array}\right.$ & $\begin{array}{l}\text { Air } \\
\mathbf{N}_{\mathbf{2}} \\
\text { Air }\end{array}$ & $\begin{array}{r}\mathbf{3 5} \\
\mathbf{0} \\
\mathbf{1 3}\end{array}$ \\
\hline
\end{tabular}

To exclude air, the tubes holding the samples were put in a vessel which was exhausted and refilled with $\mathrm{N}_{2}$ several times. The process of exhausting the air probably does not affect infectivity because at least $6 \mathrm{hr}$. without air are needed for significant inactivation, which is not increased by repeated exhaustion with final admission of air for the period of incubation. Contaminants in the $\mathbf{N}_{2}$ are probably not involved because the phenomenon appears with specially purified $\mathrm{N}_{2}$, with $\mathrm{H}_{2}$, and even when the refilling with inert gases is dispensed with but the exhaustion is prolonged. The possibility that anaerobic bacteria are concerned can probably be excluded because inactivation proceeds in $2 \mathrm{~g}$. azide/l. and in the presence of $\mathrm{CHCl}_{3}$. We report these 
results with some trepidation because it is clear that some important factor is uncontrolled; this is unsatisfactory, but does not affect the fact that inactivation of this type often happens.

\section{Miscellaneous inactivating agents}

The effect of various substances on the infectivity of TMV nucleic acid was studied in the hope of finding an environment in which infectivity was stabilized, or that a reaction would be discovered characteristic of a certain type of structure which would give information about the nature of the infective unit. Neither aim was achieved but a few observations are worth recording.

As already noted, $\mathrm{Fe}$ inactivates; $\mathrm{Zn}, \mathrm{Cu}, \mathrm{Ni}$, and $\mathrm{Mn}$ at comparable concentrations do not. Their effect depends on the environment in which they are tested. Thus $\mathrm{Zn}$ has less effect in $\mathbf{0 . 0 1} \mathrm{M}$-citrate than in water, pyrophosphate or $\mathrm{NaCl}$, but we have not been able to draw any general conclusions from these differences. $\mathrm{Ca}, \mathrm{Mg}$ and $\mathrm{Sr}$ also inactivate preparations free from citrate, but after $2 \mathrm{hr}$. at $18^{\circ}$ inactivation is extensive only in solutions containing these ions at $0.01-0.1 \mathrm{M}$; ions such as $\mathrm{Na}$ and $\mathrm{K}$ do not inactivate significantly at this concentration. This type of inactivation is unlikely to have any physiological significance.

Heparin and polygalacturonic acid sulphonate, which inhibit ribonuclease and a few other enzymes (Vandendriessche, 1956; Kaiser \& Winkler, 1955), inhibit the activation of nucleic acid by added ribonuclease, but they do not stabilize it against spontaneous inactivation. The commonly used enzyme inhibitors, azide, 8-hydroxyquinoline, iodoacetic acid and diethyldithiocarbamate, likewise had no consistent stabilizing effect. Neither spontaneous inactivation nor inactivation by ribonuclease is particularly sensitive to $\mathrm{pH}$ in the range $5-8$.

There are many analogies between the inactivation of TMV nucleic acid and of the Rothamsted strain of tobacco necrosis virus, which is sensitive to dilute solutions of formaldehyde, phenylacetaldehyde and phenylglyoxal (Bawden \& Pirie, 1957b). We have studied the effect of these agents on TMV nucleic acid and confirm Staehlin's (1958) observation that it is much more sensitive to formaldehyde than is intact TMV. Staehlin, using labelled formaldehyde, found that it combined with synthetic polynucleotides; this type of experiment does not therefore give any evidence about the possible role of structures other than nucleotides in infective nucleic acid.

Several bases were used as controls in experiments on the inactivation of nucleic acid by thiaminase in the presence of thiamine and were found to have little effect. Dr R. Markham told us of his (unpublished) observations on the presence of bases related to spermine in preparations of TMV, so, although there is no other evidence for the presence of such bases in higher plants, they seemed to merit trial as inactivators. After exposure for an hour at $20^{\circ}$ to $1 \mathrm{mM}$ spermine $\left(\mathrm{NH}_{2}\left(\mathrm{CH}_{2}\right)_{3} \mathrm{NH}\left(\mathrm{CH}_{2}\right)_{4} \mathrm{NH}\left(\mathrm{CH}_{2}\right)_{3} \mathrm{NH}_{2}\right)$ the number of lesions given by nucleic acid was $5 \%$ of the control, and even when the test was made immediately after mixing the number was down to less than half. At $0.8 \mathrm{~mm}$ inactivation is considerable and detectable at $0.1 \mathrm{~mm}$. Inactiva- 
tion is to some extent prevented by salts; $0.8 \mathrm{M}$-sodium acetate or $\mathrm{NaCl}$ diminish it and there is little inactivation in $0.02 \mathrm{M}$-citrate. In neutral salt-free solution spermine precipitates TMV nucleic acid as it does yeast nucleic acid (Razin \& Rozansky, 1959); this precipitation may be useful in fractionating nucleic acids. This precipitation is prevented by salts but the concentration needed to prevent precipitation under these conditions is only $0.1 \mathrm{M}$ which, with acetate, is too little to prevent inactivation; furthermore the precipitate formed in salt-free solution dissolves when salts are added, but infectivity is not thereby restored. Precipitation alone does not therefore account for the phenomenon. The simpler base, spermidine $\left(\mathrm{NH}_{2}\left(\mathrm{CH}_{2}\right)_{3} \mathrm{NH}\left(\mathrm{CH}_{2}\right)_{4} \mathrm{NH}_{2}\right)$ also precipitates and inactivates nucleic acid, but less powerfully and $5 \mathrm{~mm}$ is needed to get substantial inactivation in an hour. The still simpler base, putrescine $\left(\mathrm{NH}_{2}\left(\mathrm{CH}_{2}\right)_{4} \mathrm{NH}_{2}\right)$, has no effect under these conditions.

Spermine and spermidine are widely distributed in animal tissues and in yeast so that in some organisms inactivation of this type may be of physiological significance. However, it is unlikely that it does more than provide another example of what may take place in the leaf.

\section{DISCUSSION}

Our results emphasize the fragility of nucleic acid preparations made from TMV and provide ample reason for their small infectivity as compared to the intact virus. These results give no direct evidence about the nature of the minimum infective unit, but they suggest that the idea that this unit is nucleic acid may entail some redefinition of that term. If the simple proposition is accepted that tobacco mosaic virus contains only two components, nucleic acid and protein, then infectivity is clearly a property of the nucleic acid, which is protected or stabilized by the protein. However, if nucleic acid is defined more rigidly, not simply by general physical properties, but chemically as a macromolecule containing only nucleotides that are phosphoric esters of ribose or deoxyribose linked to either a purine or pyrimidine, then it cannot be stated confidently that nucleic acid alone is infective. The difficulty arises because chemical analyses and physical criteria of homogeneity cannot establish, as they can with small molecules, that preparations of nucleic acid do not contain, say $1 \%$ of some impurity. Analyses may exclude postulated contaminants if these are sufficiently characteristic types of material, but the general proposition that a nucleic acid is more than $99 \%$ pure cannot be established. This being so, it is important to consider reasons for supposing that $1 \%$ or less of the material may be as significant as the bulk of the preparation for understanding the process of infection. This is an obvious possibility when preparations are poorly infective. Even with preparations of intact TMV many thousands of particles are needed to get a lesion by current methods of inoculation, and nucleic acid is much less infective than intact virus. Hence the observed infectivity could be accounted for by postulating that only one particle in a million carries all the components necessary to make it infective. Although this may be an extreme interpretation, while it is a possible one, 
physical and chemical measurements are clearly irrelevant to identifying what may be essential components, for the precision of the measurements may be out by a factor of a thousand or more.

The history of work with TMV provides many examples of the need for caution in drawing conclusions about the nature of the infective unit and the significance of its various parts. A cautious approach to the evidence will also help to ensure that the right problem is being studied. The nucleic acid, which today gets so much attention, was at first overlooked, when TMV was described as a globulin (Stanley, 1935), and then dismissed as a contaminant (Stanley, 1937). Even after our evidence (Bawden \& Pirie, 1937) for the presence of nucleic acid was generally accepted, analytical work still centred on the protein and claims were made that 'mutations' involved changes in amino acid constitution (Stanley, 1943) and that reversible changes in the protein destroyed and restored infectivity (Ross \& Stanley, 1938). It is understandable enough that most attention should be paid to the quantitatively greater component, particularly when many people thought specificity was the prerogative of proteins, but it has long been obvious that, although nucleic acids do not offer such fantastic opportunities for specificity as proteins, they offer enough for any conceivable needs (Bawden \& Pirie, 1953).

Popular fashion has now so changed that much effort is being put into attempts to explain specificity in terms of patterns along chains containing only four nucleotides. Ironically, while this is being done, the number of nucleic acid components is steadily increasing, partly because techniques of separating them have improved, but mainly because biochemists are no longer obsessed with the idea that there are only four and so look for more. Two sugars (Smith \& Dunn, 1959) and eight bases are now recognized in normal ribose nucleic acids (Littlefield \& Dunn, 1958); others can be introduced by supplying organisms with suitable precursors and further research will no doubt reveal still more. It is too early to draw any conclusions about the part played by these other components, but their existence suggests that the effort being put into explaining heredity and some other forms of specificity on the basis of rhythms built from only four nucleotides may be misdirected. The Morse code and most computers work with only two units, but there is no reason for those who are trying to apply 'information theory' to biological synthesis to assume that Nature is similarly parsimonious. In a particle as large as TMV nucleic acid there are obviously ample opportunities for rearrangements among the nucleotides, and it has been apparent since Berzelius introduced the concept of isomerism, 120 years ago, that the arrangement of components is one important part of chemical specificity. However, arrangements may not be everything; specificity may equally depend on exactly what is arranged, and it is important to look beyond recognized nucleotides to see whether specificity, or 'information', may not be carried by other kinds of substance.

There is no a priori reason why differences between infective and noninfective nucleic acid should always depend on a break or other change in a chain consisting solely of nucleotides, rather than on a change in some 
prosthetic group or component, such as carbohydrate, lipid or peptide, attached to it. We have already argued that such components could exist without being detected by current analytical tests used to assess purity and, that being so, the problem needs to be approached indirectly, by finding whether inactivation can always be explained in terms of what is now thought to be the structure of nucleic acid. When it cannot, two courses are open. Comparable treatments can be applied to other nucleic acids or, preferably, synthetic polynucleotides (Grunberg-Manago \& Ochoa, 1955), to see whether they produce any changes that might explain the inactivation by affecting nucleotide structure or arrangement. Secondly, the concept of nucleic acid can be broadened to accommodate the inactivation, and the idea accepted that something with the general physical and chemical properties of a nucleic acid may have attached to it a highly relevant prosthetic group. The concept is already established with proteins; haemoglobin, for example, is called simply a protein, but it is the haemin and not the part of the particle composed of amino acids that is usually held responsible for its behaviour in the presence of $\mathrm{O}_{2}$ and CO. Similarly, some of the conditions in which TMV nucleic acid inactivated in our experiments suggest that there may be important parts of the infective unit other than nucleotides.

The arrangement or constitution of the predominant protein of the virus clearly plays little or no part in determining specificity, but it is no less an essential component because of this. It is highly effective in protecting the fragile infective parts against many lethal environments. The great stability of tobacco mosaic seems to lie either in the structure of its protein or in the manner in which protein and nucleic acid are combined. Although the proteins of many other viruses do not denature any more readily, these viruses nevertheless inactivate in conditions where TMV is stable; the inactivation, for example, of the Rothamsted tobacco necrosis virus by mitochondria, gentle heat or oxidation, leaves serological activity and general physical behaviour unimpaired, suggesting that such inactivating agents act on the nucleic acid. The separated nucleic acid of TMV also inactivates rapidly with similar treatments, so that in the intact virus particles it seems better protected than is the nucleic acid of viruses with spherical particles.

There is now much circumstantial evidence compatible with the idea that virus particles fragment in the initial stages of infection. If this happens differences in the susceptibility to infection of different plants, or of different leaves of one plant, could depend on the relative abilities of the cells to inactivate the infective units when these are freed from their protecting protein. We started our experiments hoping to find, by in vitro tests with nucleic acid preparations, systems that might explain the differences in susceptibility. Although this hope was not realized, our results show that, if nucleic acid does become free in vivo, it is at hazard from a variety of potential inactivators. However, as none of the inactivating systems we used seemed adequate to explain the differences in susceptibility we encountered, we studied none in great detail, and we report our results to illustrate the possibilities rather than to demonstrate what actually happens in vivo. Our failure to get any direct 
evidence on the important problem of variation in susceptibility does not exclude this mode of approaching the problem; it may mean only that we were using virus fragments different from those into which the virus separates early in the infection process, or were exposing the fragments to the wrong inactivating systems.

We thank Dr A. Isaacs, Mr R. H. Kenten and Dr W. S. Pierpoint for supplying us, respectively, with preparations of interferon, thiaminase and leaf mitochondria.

\section{REFERENCES}

Bawden, F. C. \& Prrie, N. W. (1936). Experiments on the chemical behaviour of potato virus ' $\mathrm{X}$ '. Brit. J. exp. Path. 17, 64.

Bawden, F. C. \& Pirie, N. W. (1937). The isolation and some properties of liquid crystalline substances from solanaceous plants infected with three strains of tobacco mosaic virus. Proc. Roy. Soc. B, 123, 274.

BAwDEn, F. C. \& PIRIe, N. W. (1953). Virus multiplication considered as a form of protein synthesis. In The Nature of Virus Multiplication. Symp. Soc. gen. Microbiol. 2, 21.

Bawden, F. C. \& Pirie, N. W. (1957 a). A virus-inactivating system from tobacco leaves. J. gen. Microbiol. 16, 696.

BAwDEn, F. C. \& PiRIE, N. W. (1957 $b$ ). The activity of fragmented and reassembled tobacco mosaic virus. J. gen. Microbiol. 17, 80.

Bawden, F. C. \& Roberts, F. M. (1947). The influence of light intensity on the susceptibility of plants to certain viruses. Ann. appl. Biol. 34, 286.

Bawden, F. C. \& Roberts, F. M. (1948). Photosynthesis and predisposition of plants to infection with certain viruses. Ann. appl. Biol. 35, 418.

Fraenkrl-Conrat, H. (1956). The role of nucleic acid in the reconstitution of active tobacco mosaic virus. J. Amer, chem. Soc. 78, 882.

Fraenkel-Conrat, H. \& Singer, B. (1957). Virus reconstitution: combination of protein and nucleic acid from different strains. Biochem. biophys. Acta, 24, 540.

Gierer, A. \& Schramm, G. (1956). Infectivity of ribonucleic acid from tobacco mosaic virus. Nature, Lond. 177, 702.

Grunberg-Manago, M. \& Ochoa, S. (1955). Enzymatic synthesis and breakdown of polynucleotides; polynucleotide phosphorylase. J. Amer. chem. Soc. 77, 3165.

HrLx, R. \& Lemmans, H. (1941). Studies on iron in plants with special observations on the chlorophyll:iron ratio. Biochem. J. 35, 1190.

Holden, M. \& PIrIE, N. W. (1955). The partial purification of leaf ribonuclease. Biochem. J. 60, 39.

Isacs, A. \& Lindenmann, J. (1957). Virus interference. I. The interferon. Proc. Roy. Soc. B, 147, 258.

Kaiser, E. \& Winkier, G. (1955). Zur Frage der kompetitiven Hemmung des Hyaluronsäure-Hyaluronidasesystems. Arzneim.-Forsch. 5, 322.

KassanIs, B. (1952). Some effects of high temperature on the susceptibility of plants to infection with viruses. Ann. appl. Biol. 39, 358.

Kreten, R. H. (1957 a). Bracken thiaminase. Rep. Roth. exp. Sta. 1957, 242.

Kenten, R. H. $(1957 b)$. The partial purification and properties of a thiaminase from bracken. Biochem. J. 67, 25.

KLEczKowski, A. (1950). Interpreting relationships between the concentrations of plant viruses and numbers of local lesions. J. gen. Microbiol. 4, 53.

Knight, C. A. (1956). Preparation of ribonucleic acid from plant viruses. Methods in Enzymology, 3, 684. 
LitTlefieLd, J. W. \& DunN, D. B. (1958). The occurrence and distribution of thymine and three methylated adenine bases in ribonucleic acids from several sources. Biochem. J. 70, 642 .

Loring, H. S., AL-RAwi, S. \& Fujimoto, Y. (1958). The iron content of tobacco mosaic virus and some properties of its infectious nucleic acid. J. biol. Chem. 233,1415 .

Pierpoint, W. S. (1959). Mitochondrial preparations from the leaves of tobacco (Nicotiana tabacum). Biochem. J. 71, 518.

Pirie, N. W. (1956). Some components of tobacco mosaic virus preparations made in different ways. Biochem. $J .63,316$.

Pirie, N. W. (1959). Leaf proteins. Annu. Rev. Plant Physiol. 10, 33.

Razin, S. \& Rozansky, R. (1959). Mechanism of the antibacterial action of spermine. Arch. Biochem. Biophys. 81, 36.

Ross, A. F. \& Stanley, W. M. (1938). The partial reactivation of formolized tobacco mosaic virus protein. J. gen. Physiol. 22, 165.

Smith, J. D. \& DUNN, D. B. (1959). An additional sugar component of ribonucleic acids. Biochim. biophys. Acta, $31,573$.

Stafhelin, M. (1958). Reaction of tobacco mosaic virus nucleic acid with formaldehyde. Biochim. biophys. Acta, 29, 410.

StanLEY, W. M. (1835). Isolation of a crystalline protein possessing the properties of tobacco mosaic virus. Science, 81, 644 .

Stanley, W. M. (1987). Chemical studies on the virus of tobacco mosaic. VIII. The isolation of a crystalline protein possessing the properties of aucuba mosaic virus. J. biol. chem. 117, 325.

Stanley, W. M. (1943). Chemical structure and the mutation of viruses. In Virus Diseases, p. 85. Ithaca, New York: Cornell University Press.

VANDENDRIessche, L. (1956). Inhibitors of ribonuclease activity. Arch. Biochem. Biophys. 65, 347.

(Received 27 April 1959) 